\title{
Design Considerations for Web-Based Interactive TV Services*
}

\author{
Meng-Huang Lee and He-Rong Zhong \\ Department of Information Management, Shih-Chien University, Taipei, 104 Taiwan \\ meng@mail.usc.edu.tw
}

\begin{abstract}
Due to the performance limitation of set-top-box and viewing behavior of TV users, web page design for ITV services is not as same as for Internet. In this paper, we present two practical concerns and their solutions for the web application design for web-based ITV services.
\end{abstract}

\section{Introduction}

Current interactive TV (ITV) services adopt web-based and IP based technologies [1]. In web-based ITV services, there is a set-top-box connecting to Internet by ADSL or Cable Modem and using TV monitor as its display device [4]. By these technologies, ITV services are very similar to traditional Internet services except the devices at client's side are set-top-box with TV display. For the technical issues and cost issues, a set-top-box is something like a PC but with limited resources. It also has CPU, RAM, small disk size and MPEG-2 decoder, etc. but the computing power and capacity are far away from the specifications of current off-the-shelf PC [2],[3].

In addition to system resource limitation, ITV user's viewing behavior issue also makes the web page design different from Internet. For computer users, the input or navigation devices are keyboard and mouse. When they click one button or keystroke, they usually watch the response from the computer. But for ITV users, they use controllers as their interactive devices and they are used to the quick response of traditional TV services. In web-based ITV services, the browsing and navigation procedure are similar to Internet web-based services. The system loads the web pages from web servers and the browser in the set-top-box then processes web pages and displays the results on TV screen. For the limited processing power of set-top-box, the response time of web page transition is not as quick as expectation of traditional TV services. Most ITV users do not know these procedures, they just wonder if something wrong in the system. Then a lot of controller presses are issued and finally the set-top-box crashes. Turn-off the set-top-box and turn-on again seems the only way that users can do for the situations.

In this paper, we propose two practical solutions in our system design. In section 2, key-lock mechanism is used to disable all the inputs during the web page transition and avoids system crash. In section 3, scrolling mechanism is used to reduce the number of web transition of an ITV service.

This work has been supported in part by Shih-Chien University, Taiwan, under Grant USC 93-05-38804-035 and by Imagetech Co. Ltd, Taipei, Taiwan 


\section{Key-Lock During Web Page Transition}

The web page transition usually occurs when a page is leaving and a new page is loading. During the transition, the system design is to disable all the inputs from users and make sure the transition is safe. To assure the key-lock during the transition, keylock mechanism is activated when current page is leaving and the new page is fully loaded. Fig.1 shows the key-lock mechanism for the web page transition.

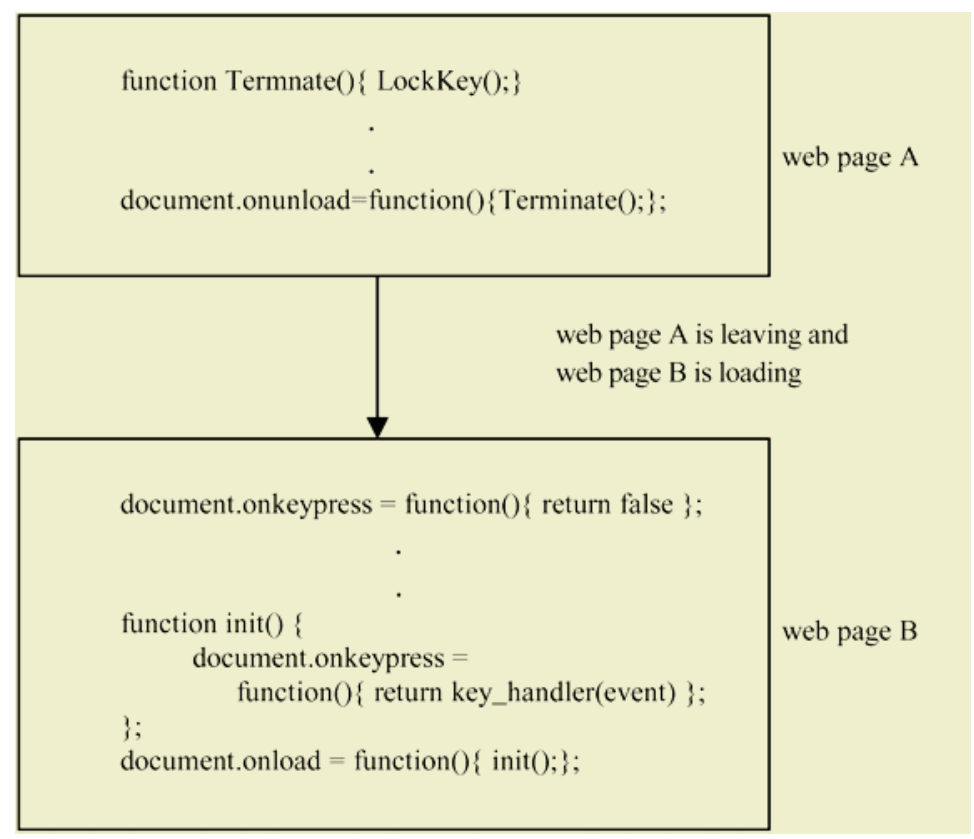

Fig. 1. The key-lock mechanism during web page transition

\section{Using Scrolling Mechanism to Reduce Number of Web Page Transition}

Due to the slow transition of web pages, avoiding lots of web page transition in a service is a key design concept. In traditional web applications, the browser can resize its display window, and if the page presentation exceeds the size, then a scroll bar in horizontal axis or vertical axis will appear such that the user can scroll the presented page. But for ITV services, TV screen resolution is fixed (e.g. NTSC screen is set to 640X480) and it lacks the input devices (e.g. mouse) for a user scrolls the presented page manually so that the exceeded part of the presented page can not be seen on screen by user. This feature gives a good direction for the web page design to avoid lots of web page transition. Fig. 2 assumes three menu items and an area on the right side to show the corresponding information page when a menu item is selected. According our design described above, the information pages for Menu-item-1, Menuitem-2, and Menu-item-3 are all loaded in one web page transition. If a user selects Menu-item-2, our web page program just scrolls to the corresponding position of the 
information page of Menu-item-2. For the TV resolution is fixed, only Menu-item-2 information page can be seen on the screen and information pages of Menu-item-1 and Menu-item-3 are hidden on the screen(even though they are loaded).

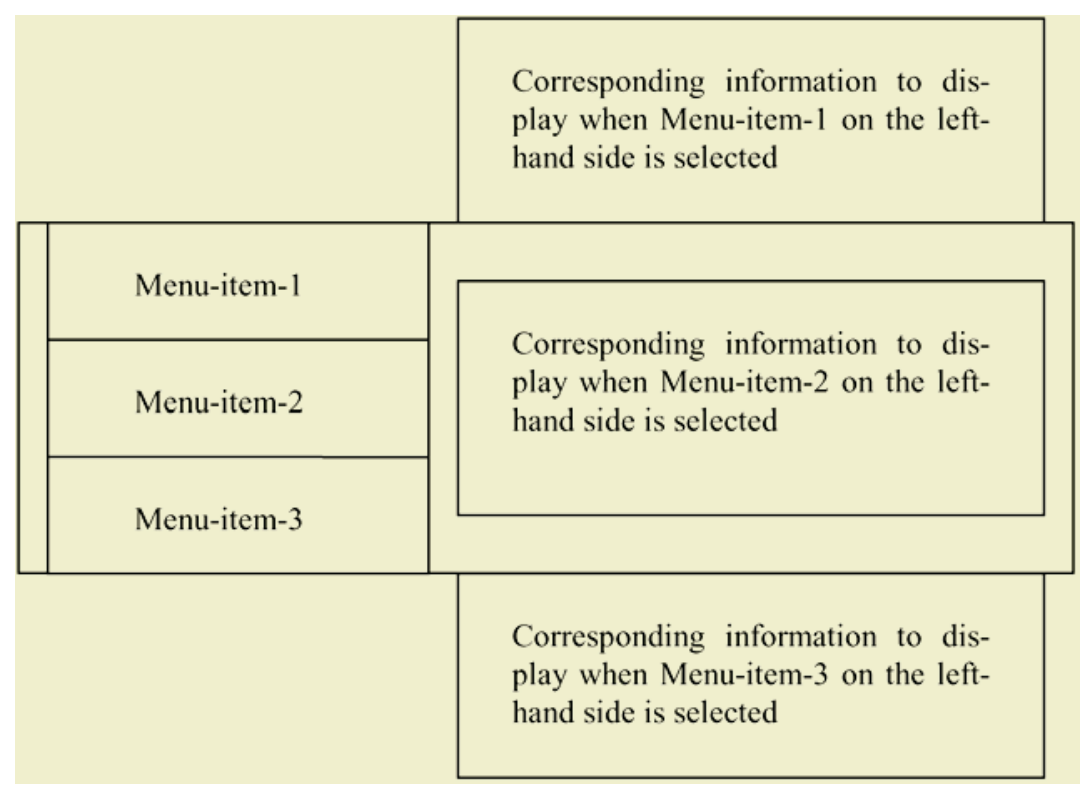

Fig. 2. An example for the menu application using scrolling design

\section{References}

1. George Abe: Residential Broadband, Second Edition.Cisco Press, 2000.

2. Browser Development Guide. 2000 Pace Micro Technology plc.

3. Web \& Video Application Design's Guide for IP Set-Top-Box (PB03018 Project). Foxconn, 2004.

4. Meng-Huang Lee: System Architecture for Interactive Home TV Services. $8^{\text {th }}$ IEEE International Symposium on Consumer Electronics, Reading, UK, September 3, 1996. 